\title{
The psychological impact and experience of breast cancer screening in young women with an increased risk of breast cancer due to neurofibromatosis type 1
}

\author{
Ashley Crook ${ }^{1,2}$ D $\cdot$ Rebekah Kwa $^{3} \cdot$ Sarah Ephraums $^{3} \cdot$ Mathilda Wilding $^{1,2} \cdot$ Lavvina Thiyagarajan $^{1}$. \\ Jane Fleming $^{1}$ (D) $\cdot$ Katrina Moore ${ }^{4} \cdot$ Yemima Berman $^{1}$ (D)
}

Received: 24 March 2021 / Accepted: 20 April 2021 / Published online: 8 May 2021

(c) Crown 2021

\begin{abstract}
Women with neurofibromatosis type 1 (NF1) have an increased risk of developing early breast cancer with a poorer prognosis compared to the general population. Therefore, international management guidelines recommend regular screening in women with NF1 starting from 30 to 35 years. As the psychological impacts of breast cancer screening in other high-risk populations cannot be extended to women with NF1, due to increased incidence of cognitive and mental health issues, the psychological harms of breast screening in women with NF1 are unknown. Consequently, the aim of this study was to assess the psychological impact of breast cancer screening in women with NF1 attending an established risk management clinic. Twenty-eight women with NF1 (30-50 years) completed psychological well-being and patient experience questionnaires, administered across five time points, before and after their initial and second round annual breast screening visits. Preliminary findings demonstrated the screening regimen was well-tolerated, with most participants reporting high satisfaction with the screening process. Overall, no significant increase in psychological distress related to the breast screening process was identified, with mean cancer worry and anxiety scores decreasing over time. However, some women did experience negative aspects of screening and barriers to re-attendance at annual breast screening appointments. As some women with NF1 exhibited clinical levels of psychological distress prior to screening, efforts to identify those at risk and additional support to address concerns and expectations throughout the breast screening process may be beneficial.
\end{abstract}

Keywords Breast cancer $\cdot$ Neurofibromatosis type $1 \cdot$ Screening $\cdot$ Psychosocial impact $\cdot$ Experience and barriers

\section{Introduction}

Women with Neurofibromatosis type 1 (NF1) have a fivefold increased risk of developing breast cancer by age 50 [1-3] and recent guidelines recommend annual breast

Ashley Crook

Ashley.Crook@health.nsw.gov.au

1 Department of Clinical Genetics, Royal North Shore Hospital, Sydney, NSW, Australia

2 NSLHD Familial Cancer Service, Department of Cancer Services, Royal North Shore Hospital, Sydney, NSW, Australia

3 Northern Clinical School, Faculty of Health and Medicine, University of Sydney, Sydney, NSW, Australia

4 Department of Breast Surgery, Royal North Shore Hospital, Sydney, NSW, Australia screening from age 30 [4, 5] or 35 [6]. In comparison to other inherited breast cancer predisposition conditions, individuals with NF1 have a high incidence of co-morbidities, cognitive deficits and mental health problems, which may complicate the screening process. Given there is a paucity of evidence regarding the patient experience and psychological impact of breast screening for women with NF1, this is an important area of further exploration.

NF1 is a tumour susceptibility syndrome that predisposes affected individuals to cutaneous, subcutaneous and plexiform neurofibromas, malignant peripheral nerve sheath tumours, and central nervous system tumours [7-9]. The condition displays autosomal dominant inheritance, with complete penetrance but variable phenotypic expressivity [10]. Lisch nodules, axillary and inguinal freckling, and café au lait spots are characteristic features [11]. There are several other health concerns associated with NF1 including chronic pain and itch, skeletal disorders, muscle weakness, 
cardiovascular abnormalities, neurocognitive deficits and increased psychiatric morbidity, which require multidisciplinary input [7, 12-18]. Cognitive deficits normally manifest as mild intellectual impairment, learning difficulties or attentional difficulty $[14,19]$, with $8 \%$ of individuals with NF1 intellectually disabled [19]. As NF1 impacts physical, cognitive and psychological functioning, it has been reported to have an adverse effect on quality of life [20]. It is also associated with higher rates of anxiety and depression than the general population, with anxiety and/or depressive symptoms identified in more than half of those from two adult NF1 cohorts [18, 21].

In the past 15 years, evidence for breast cancer risk in women with NF1 has increased [1-3, 8, 9, 13, 22-28]. While lifetime risk of developing breast cancer is moderately increased (18\%) [3], women with NF1 aged under 50 have a five-fold increased risk of developing breast cancer and present with more advanced disease compared to the general population [2]. Other studies have identified that women with NF1 have an elevated breast cancer mortality and poorer breast cancer survival [3, 9, 28, 29]. A possible reason for the poorer breast cancer survival rates in women with NF1 (67.9\% 5-year survival compared to $87.8 \%$ in the general population) is the overrepresentation of poorer prognostic tumour characteristics, which are associated with more advanced-stage presentations [2, 3]. In addition, a diagnosis may be delayed if patients with NF1 have lower health activation [30, 31]. It has also been hypothesized that time to diagnosis may be prolonged due to difficulty differentiating malignant tumours from neurofibromas on clinical breast examination as well as on mammography [28, 29].

Current Australian guidelines recommend annual breast screening for women with NF1 from the age of 35, and this includes magnetic resonance imaging (MRI), \pm mammogram \pm ultrasound [6]. Internationally, the starting age may begin from age $30[2,4,5]$. The potential harms associated with breast cancer screening, identified in other high risk cohorts, include pain or discomfort [32], false positive results [32-34], radiation exposure [32], over diagnosis [32, 35], and psychological issues such as anxiety, cancer worry, or other forms of distress that may be related to screening itself, or needing to be recalled for review or biopsy [32-34, 36-40]. This can negatively impact on future screening attendance, meaning patients do not access the benefits of regular screening $[32,37]$. Although screening-related worry is mostly transient [34, 35, 38-40], distress may persist in those with high baseline anxiety $[34,38]$ or with a family history of breast cancer $[34,36]$, although this is not consistent in all studies [38].

Despite the possible harms, studies of other populations at increased risk of developing breast cancer, such as carriers of $B R C A 1$ or $B R C A 2$ pathogenic variants, suggest the benefits of screening far outweigh the harms [35, 36, 38-47]. A psychological benefit is also seen in TP53 pathogenic variant carriers, who have another multiple tumour-prone syndrome, Li Fraumeni syndrome (LFS) [48]. These findings cannot be extrapolated to the NF1 population due to the additional psychosocial characteristics associated with this condition. In order to understand the psychological impact of breast cancer screening over time and explore the experiences and perceived barriers to screening uptake, we prospectively surveyed young women with NF1 (30-50 years) as they enrolled in a high-risk breast cancer screening clinic.

\section{Materials and methods}

This is a pilot, prospective longitudinal cohort study conducted through a tertiary hospital adult NF1 clinic and an established breast cancer risk management clinic in Sydney, Australia. The study involved three sub-studies. This paper will report on the patient experiences and psychological impact of breast screening and cancer discussions. The other sub-studies (breast cancer screening outcomes and feasibility and the development and evaluation of patient education resources) will be reported separately.

\section{Participants}

As part of the overall study, women (30-50 years) with clinically diagnosed NF1 [11] were recruited from the hospital's adult NF1 clinic to attend the breast cancer risk management clinic for annual breast screening (which may include breast MRI, mammogram, ultrasound or biopsy). Participants were eligible to enrol in the risk management clinic if they fulfilled the following criteria: previous or current referral to the hospital's NF1 clinic, an ECOG status of 0 or 1; no active cancer diagnosis; no previous breast cancer diagnosis; not currently pregnant; and, an expected lifespan greater than three years. Prior to being referred to the clinic, potentially eligible participants were first informed of the breast cancer risk in NF1, screening options, screeningassociated risks and uncertainties related to the benefits of screening younger women with NF1. Socio-demographic data were also collected at baseline through patient interview and medical record review. This occurred either at their routine NF1 management appointment, or by telephone.

Individuals who were invited to screening were also invited to participate in this sub-study, which involved the provision of written consent. Those who attended for screening were asked to complete questionnaires at different time points before and after their initial and second round annual breast screening visits. Those who declined their first or second round screening were also invited to complete a questionnaire. Women were excluded from this sub-study if they 
were unable to provide informed consent, undergo study procedures or understand an English language consent form.

\section{Instrumentation}

Three questionnaires were developed, which were administered at five separate time points. Questionnaire 1 (Q1) comprised several scales used to measure anxiety and depression (Hospital Anxiety and Depression Scale, HADS) [49] and the 6-item State-Trait Anxiety Inventory, STAI-6 [50-52]), cancer worry (adapted Cancer Worry Scale, CWS [53, 54]); and behaviour changes (Health Questionnaire, HQ) [55]). HADS is a 14-item scale used to measure anxiety and depression over the preceding week. Scores $>11$ points indicate clinically significant anxiety and/or depression while scores between 8 and 10 indicate borderline anxiety and/or depression [49]. The 6-item STAI questionnaire is a modified short-form of the original 40 item STAI questionnaire and is a validated measure of anxiety for current state of mind and situational factors that may influence anxiety levels: with scores $>36$ indicating high anxiety [50-52] (partial set of STAIAD items used with permission of the publisher. STAIAD instrument ( 1968,1977 Charles D Spielberger. All rights reserved in all media. Published by Mind Garden, Inc., www.mindgarden.com). The CWS measures cancer related worry and its impact in an 8-item questionnaire with each question scored on a 4-point Likert scale. A score $>14$ indicates severe cancer worry while a score $>12$ can be used as a screening measure for possible distress (range 4-24) $[53,54]$. The HQ measures perceived stress related behaviours in the week prior to taking the questionnaire with a score of ' 0 ' indicating "better than normal behaviour"; a score of " 1 " indicating "normal behaviour" and a score of "2" indicating "worse than normal behaviour" with scores previously shown to be correlated with HADS anxiety and depression levels [55].

Questionnaire 1 for decliners (Q1D) included all measures above plus an additional question regarding participant's reason for declining breast screening. Questionnaire 2 (Q2) included the measures outlined above plus ad hoc scales to assess MRI, mammography and ultrasound experience (a 6-item, 5-point Likert scale), barriers (a 5-item, 5 -point Likert scale) and satisfaction (1 item, a 5-point Likert scale), which were adapted from an existing protocol for a surveillance study on multi-organ cancer prone syndromes [56] (Online Resource 1). A free text space for comments on experience of each screening modality was also provided.

\section{Procedures}

For those who completed breast screening, questionnaires were distributed to participants at four time points ( $\mathrm{T} 1$, $\mathrm{T} 2, \mathrm{~T} 3, \mathrm{~T} 4)$ in the first round (R1) and one time point after completion of the second round of breast screening (R2). Q1 was provided at T1, T2 and T4 in R1. Q2 was provided at $\mathrm{T} 3$ in $\mathrm{R} 1$ and at $\mathrm{R} 2$. Those who had declined either R1 or R2 screening were forwarded Q1D at a single time point (Table 1). Questionnaires were sent or provided directly to the participant at their hospital visit. Participants completed the questionnaires on paper or using an individualised link to an online REDCap (Research Electronic Data Capture) survey [57]. Participants were

Table 1 Study design — timeline of completion of questionnaires

\begin{tabular}{|c|c|c|c|}
\hline Breast screening process & $\begin{array}{l}\text { Planned timeframe for Questionnaire } \\
\text { completion }\end{array}$ & Time point & Questionnaire \\
\hline \multicolumn{4}{|l|}{ ROUND 1 (R1) } \\
\hline Recruitment to study & At recruitment & Time point 1 (T1) (Baseline) & Q1 (HADS, HQ, CWS, STAI-6) \\
\hline Breast screening & $7-14$ days after recruitment ${ }^{\mathrm{a}}$ & Time point 2 (T2) & Q1 (HADS, HQ, CWS, STAI-6) \\
\hline \multirow{2}{*}{$\begin{array}{l}\text { Appointment to dis- } \\
\text { cuss breast screening } \\
\text { results }\end{array}$} & 7-14 days after results appointment & Time point 3 (T3) & $\begin{array}{l}\text { Q2 (HADS, HQ, CWS, STAI-6, experience } \\
\text { of and barriers to screening) }\end{array}$ \\
\hline & $6-12$ weeks after results appointment & Time point 4 (T4) & Q1 (HADS, HQ, CWS, STAI-6) \\
\hline \multicolumn{4}{|l|}{ ROUND 2 (R2) } \\
\hline $\begin{array}{l}\text { Breast screening and } \\
\text { appointment to } \\
\text { discuss results }\end{array}$ & 6-12 weeks after results appointment & Round 2 (R2) & $\begin{array}{l}\text { Q2 (HADS, HQ, CWS, STAI-6, experience } \\
\text { of and barriers to screening) }\end{array}$ \\
\hline \multicolumn{4}{|l|}{ DECLINERS } \\
\hline & $\begin{array}{l}\text { 6-12 weeks after scheduled screening } \\
\text { date in } R 1 \text { or } R 2\end{array}$ & & $\begin{array}{l}\text { Q1D (HADS, HQ, CWS, STAI-6, reason } \\
\text { for declining) }\end{array}$ \\
\hline
\end{tabular}

HADS Hospital Anxiety and Depression scale, HQ Health Questionnaire, CWS cancer worry scale; and STAI-6 6-item State Trait Anxiety Inventory

${ }^{a}$ All T2 questionnaires were completed pre-results appointment, however, due to clinical timelines some were completed pre-screening and some post-screening 
followed up at least twice either by email, SMS text message, by telephone or at their scheduled appointment if they had not completed their questionnaire within the initial scheduled completion time.

\section{Analysis}

Demographic information, baseline characteristics, the ad hoc screening experience and reasons for declining are reported using descriptive statistics only. Outliers were removed and due to missing data some results are reported according to the denominator. Paired-t tests were used to assess changes in psychological test scores over time, Pearson's Chi-squared test was employed to measure any associations between demographic factors and screening-related distress (data was dichotomized into age 30-34/age 35-50; family history/no family history of cancer in a first degree relative; recall/no recall and biopsy/ no biopsy) with Fisher's exact test applied when expected cell count was low $(<5$ in a cell) and McNemar's test utilized to assess any change in the proportion of subjects in each category (normal, borderline, abnormal) at baseline compared to follow-up. For analysis, ages were dichotomized into two groups 30-34 and 35-50 years. This was a decision based on two reasons: (1) we were interested in whether extending our local screening guidelines to younger women between ages 30-34 would be associated with increased psychological distress; (2) we had a relatively young cohort (mean age 35.61 at T1) and therefore there were not enough women in a 40-49 year age group for analysis. Analysis was performed using IBM SPSS Statistics for Windows, Version 25.0. IBM SPSS Statistics Version 25. Armonk, MY: IBM Corp.

\section{Results}

Forty-eight women were invited to undergo breast screening through the risk management clinic (recruitment commenced July 12, 2018). As of 12 February 2021, 36 women had enrolled in the risk management clinic. Reasons why 12 women were not enrolled included: unable to contact to discuss participation $(n=3)$, declined and no reason provided $(n=3)$, declined due to time required for appointments and/ or associated travel $(n=3)$, declined as already accessing breast screening $(n=3)$.

Twenty-eight women agreed to participate in this substudy (response rate $78 \%$ ) (mean age $35.61 \pm 4.47$; range 30-47 years). Nine (32.1\%) had previously had breast screening, although none had previously had MRI breast screening (Table 2). Twenty-two of the participants (mean age: $36.90 \pm 4.42$ ) attended a subsequent round of annual breast screening (R2). All three women who were recalled in $\mathrm{R} 2$ were also recalled in $\mathrm{R} 1$. No cases of breast cancer were seen in either the first or second round of screening.

For R1 and R2 surveys, completion times varied amongst the participants, as many participants did not complete the questionnaire in the time requested and needed to be followed up. Drop out from screening was also observed (Fig. 1).

\section{Baseline anxiety, depression, cancer worry and behavioural change}

At baseline (time point 1$)(\mathrm{n}=28)$, mean HADS anxiety and depression scores were at the top of the normal range for anxiety $(7.25 \pm 4.20)$ with $6 / 28(21.4 \%)$ participants having a borderline and 6/28 (21.4\%) having clinical anxiety scores. In comparison, mean HADS depression scores were within the normal range $(2.61 \pm 2.69)$; and $26 / 28$ (92.9\%)
Table 2 Study participant demographics

\begin{tabular}{llll}
\hline & $\begin{array}{l}\text { Overall participant } \\
\text { data } \\
\mathrm{n}(\%)\end{array}$ & $\begin{array}{l}\mathrm{R} 1 \text { screening } \\
\mathrm{n}(\%)\end{array}$ & $\begin{array}{l}\mathrm{R} 2 \text { screening } \\
\mathrm{n}(\%)\end{array}$ \\
\hline Number of participants & $28(100)$ & $27(96.4)$ & $22(78.6)$ \\
Family history of NF1 in FDR & $12(42.8)$ & $11(40.7)$ & $8(36.3)$ \\
Family history of cancer in FDR & $9(32.1)$ & $9(33.3)$ & $8(36.3)$ \\
Family history of breast/ovarian cancer & $3(10.7)$ & $3(11.1)$ & $3(13.6)$ \\
$\quad$ in FDR & $9(32.1)$ & - & - \\
Screening prior to enrolment & 0 & - & - \\
MRI & $6(21.4)$ & - & - \\
Mammogram & $8(28.6)$ & - & $3(13.6)$ \\
Ultrasound & - & $11(40.7)$ & $2(9.1)$ \\
Recall & - & $9(33.3)$ & \\
Biopsy & & & \\
\hline
\end{tabular}

$N F 1$ neurofibromatosis type $1, F D R$ first degree relative 
Fig. 1 Summary of participation in this study. ${ }^{\mathrm{a}}$ At $\mathrm{R} 2$, one additional participant submitted an incomplete questionnaire

\section{Timeline and participants}
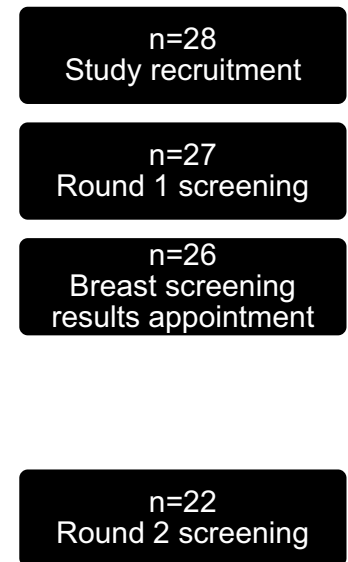

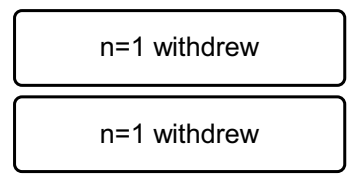

$\mathrm{n}=5$ did not complete $\mathrm{R} 2$

\section{Questionnaires completed}

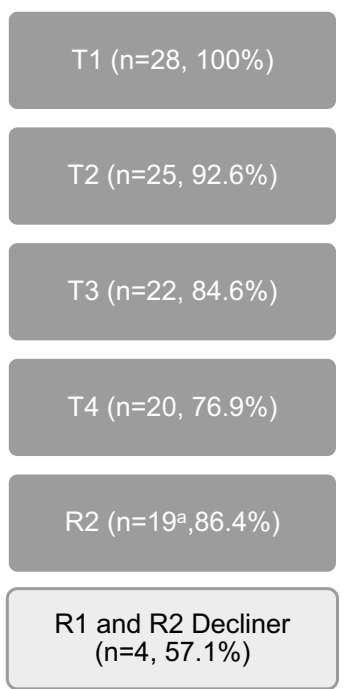

of participants had a normal depression score, the remainder exhibiting borderline depression. Mean CWS score was within the normal range $(14.86 \pm 3.58)$, however, over half reported scores indicating severe cancer worry $(60.7 \%)$. For individual questions, several participants reported frequent or constant thoughts regarding 'the future possibility of surgery' (7/28 (25.0\%)); 'the future possibility of developing cancer' (6/28 (21.4\%)), 'the chance of family members developing cancer' $(6 / 28(21.4 \%))$; 'the frequency of cancer worry' (5/28 (17.9\%)), and 'the worry was frequently or constantly a problem in their lives' $(5 / 28(17.9 \%))$. The mean HQ score was within the normal range $(8.04 \pm 1.86)$ where fifteen participants (53.6\%) had an average score indicating no change or improving behaviours and 13 (46.4\%) reported worsening behaviours in the week prior to completing the questionnaire. The mean general state anxiety (STAI-6) score was in the high anxiety range, $39.52 \pm 11.4$ (range 20-57). Whilst 9/28 (32\%) reported no change or low anxiety, 19/28 (68\%) exhibited high levels of state anxiety (how one feels at the moment).

\section{Psychological impact of round 1 screening}

There was no significant change in mean scores for HADS anxiety and depression, and HQ between baseline (T1) and pre- (T2) or post-screening results (T3 and T4) (Tables 3 and 4). In addition, no significant difference was observed in the proportion of women falling into the categories of clinical concern at baseline compared to follow-up in any of the scales (Online Resource 2). However, there was a significant decrease in cancer worry score from baseline (T1) to postbreast screening (T4) ( $\mathrm{z}$ score $-2.077, \mathrm{p}=0.038)$ and a reduction in STAI-6 anxiety scores T1 to T4 of 4.93 points $(\mathrm{t}=2.167, \mathrm{df}=18, \mathrm{p}=0.044)$ (Table 3$)$. In addition, analysis of mean single-item scores at baseline (T1) compared to follow-up (T4) demonstrated three potentially important aspects of screening. For CWS (question 1), 'thoughts about the chance of getting cancer' were significantly decreased $(\mathrm{z}=-2.236, \mathrm{p}=0.025)$; in HQ, women demonstrated significantly reduced irritability $(\mathrm{t}=2.517, \mathrm{df}=19, \mathrm{p}=0.021)$ and in the STAI-6 scale, participants exhibited a significantly increased feeling of calm $(\mathrm{t}=2.373, \mathrm{df}=19, \mathrm{p}=0.028)$. Individual scores also indicated a proportion of participants exhibit clinically relevant anxiety, depression, cancer worry, and change in health behaviours at baseline and throughout the screening process (Online Resource 3).

\section{Factors impacting anxiety, depression, cancer worry and behaviour}

Post-enrolment and pre-results (T2), older women (35-50 years) were significantly more likely to record worse stress-related behaviours in the previous week compared to younger women (30-34 years) (76.9\% vs 33.3\%) $\left(\chi^{2}(1)=4.812, \mathrm{p}=0.028\right)$. The most frequently reported adversely affected behaviours in older women were sleep (28.6\%), being able to stop worrying (28.6\%), irritability (28.6\%), ability to relax (21.4\%) and ability to concentrate $(21.4 \%)$. In addition, participants with a family history of any cancer in a first degree relative $(33 \%)$ were 
Table 3 Hospital Anxiety and Depression, Cancer Worry, Health Questionnaire and 6-item State Trait Anxiety Inventory mean scores over time in round 1 screening

\begin{tabular}{|c|c|c|c|c|c|}
\hline & $\begin{array}{l}\mathrm{T} 1 \\
\mathrm{n}=28\end{array}$ & $\begin{array}{l}\mathrm{T} 2 \\
\mathrm{n}=25\end{array}$ & $\begin{array}{l}\mathrm{T} 3 \\
\mathrm{n}=22\end{array}$ & $\begin{array}{l}\mathrm{T} 4 \\
\mathrm{n}=20\end{array}$ & $\begin{array}{l}\mathrm{R} 2 \\
\mathrm{n}=19\end{array}$ \\
\hline \multicolumn{6}{|c|}{ Hospital Anxiety and Depression scores (HADS) } \\
\hline Anxiety, mean (SD) & $7.25(4.20)$ & $6.74(3.88)$ & $6.39(4.96)$ & $6.55(4.35)$ & $7.68(5.66)$ \\
\hline Normal anxiety, n (\%) & $16(57.1 \%)$ & $13(52 \%)$ & $13(61.9 \%)$ & $15(75 \%)$ & $12(63.2 \%)$ \\
\hline Borderline anxiety, $\mathrm{n}(\%)$ & $5(17.9 \%)$ & $10(40 \%)$ & $5(19 \%)$ & $2(10 \%)$ & $3(15.8 \%)$ \\
\hline Abnormal anxiety, n (\%) & $7(25 \%)$ & $2(8 \%)$ & $4(19 \%)$ & $3(15 \%)$ & $4(21 \%)$ \\
\hline Depression, mean (SD) & $2.61(2.69)$ & $3.18(3.42)$ & $3.2(3.89)$ & $2.95(3.44)$ & $3.47(4.03)$ \\
\hline Normal depression, $\mathrm{n}(\%)$ & $26(92.9 \%)$ & $22(88 \%)$ & $18(81.8 \%)$ & $18(90 \%)$ & $17(89.5 \%)$ \\
\hline Borderline depression, $\mathrm{n}(\%)$ & $1(3.6 \%)$ & $1(4 \%)$ & $2(9.1 \%)$ & 0 & $2(10.5 \%)$ \\
\hline Abnormal depression, n (\%) & $1(3.6 \%)$ & $2(8 \%)$ & $2(9.1 \%)$ & $2(10 \%)$ & 0 \\
\hline \multicolumn{6}{|l|}{ Cancer Worry Scale (CWS) } \\
\hline Cancer worry score, mean (SD) & $14.86(3.58)$ & $14.70(4.32)$ & $14.55(3.55)$ & $14.15(3.44)$ & $14.68(4.26)$ \\
\hline Normal cancer worry, n (\%) & $2(7.1 \%)$ & $3(12 \%)$ & $4(18.2 \%)$ & $6(30 \%)$ & $3(15.8 \%)$ \\
\hline Possible cancer worry, n (\%) & $9(32.2 \%)$ & $6(24 \%)$ & $5(22.7 \%)$ & $4(20 \%)$ & $5(26.3 \%)$ \\
\hline Severe cancer worry, n (\%) & $17(60.7 \%)$ & $16(64 \%)$ & $13(59.1 \%)$ & $10(50 \%)$ & $11(57.9 \%)$ \\
\hline \multicolumn{6}{|l|}{ Health Questionnaire (HQ) } \\
\hline Behaviour score, mean (SD) & $8.04(1.86)$ & $7.64(1.60)$ & $8.09(2.52)$ & $7.25(2.59)$ & $7.95(2.70)$ \\
\hline No change, $\mathrm{n}(\%)$ & $12(42.9 \%)$ & $8(32 \%)$ & $11(50 \%)$ & $11(55 \%)$ & $11(57.9 \%)$ \\
\hline Better, n (\%) & $3(10.7 \%)$ & $4(16 \%)$ & $2(9.1 \%)$ & $4(20 \%)$ & $3(15.8 \%)$ \\
\hline Worse, n (\%) & $13(46.4 \%)$ & $13(52 \%)$ & $9(40.9 \%)$ & $5(25 \%)$ & $5(26.3 \%)$ \\
\hline \multicolumn{6}{|c|}{ 6-item State Trait Anxiety Inventory (STAI-6) } \\
\hline Anxiety score, mean (SD) & $39.52(11.36)$ & $39.60(13.89)$ & $37.80(13.70)$ & $34.48(15.14)$ & $43.86(17.01)$ \\
\hline Normal, n (\%) & $9(32.1 \%)$ & $12(48 \%)$ & $9(40.9 \%)$ & $11(55 \%)$ & $5(26.3 \%)$ \\
\hline High anxiety, n (\%) & $19(67.9 \%)$ & $13(52 \%)$ & $13(59.1 \%)$ & $9(45 \%)$ & $14(73.7 \%)$ \\
\hline
\end{tabular}

$H A D S$ Normal score $\leq 7$; borderline $=8-10$; abnormal $\geq 11$ (range $0-21$ ). CWS Normal score is $<12$; a score $\geq 14$ indicates severe cancer worry while a score $\geq 12$ can be used as a screening method for possible cancer worry. $H Q$ A score of 7 indicates no change in behaviour; a score $<7$ indicates an improvement in stress-related behaviours and a score $>7$ indicates a worsening in stress related behaviours (Range 0-14). STAI-6 Normal scores are defined $34-36,<34$ represents lower levels of anxiety, and $>36$ high levels of anxiety (Range 20-80)

Table 4 Psychological impact of Round 1 breast screening

\begin{tabular}{|c|c|c|c|c|c|}
\hline & Baseline (T1) & $\mathrm{T} 4$ & Mean difference & Confidence interval & P-value \\
\hline HADS Anxiety: Mean score (SD) & $6.70(\mathrm{SD} 4.03)$ & $6.55(\mathrm{SD} 4.35)$ & .150 & $-1.65-1.95$ & .863 \\
\hline HADS Depression: Mean score (SD) & 2.55 (SD 3.09) & $2.95(\mathrm{SD} 3.44)$ & -.400 & $-1.53-.73$ & .468 \\
\hline CWS: Mean score (SD) & $15.15(\mathrm{SD} 3.88)$ & 14.15 (SD 3.44) & & & 0.038 \\
\hline HQ score: Mean (SD) & $8.00(\mathrm{SD} 2.00)$ & 7.25 (SD 2.59) & .750 & $-6.32-2.13$ & .270 \\
\hline STAI-6 score: Mean (SD) & 37.54 (SD 11.69) & 32.61 (SD 12.97) & 4.93 & $0.151-9.71$ & 0.044 \\
\hline
\end{tabular}

Hospital Anxiety and Depression Scale (HADS), Cancer Worry Scale (CWS), Health Questionnaire (HQ) and 6-item State Anxiety Trait Inventory (STAI-6). Parametric t-tests used in analysis HADS, HQ and STAI-6 results. Non-parametric Wilcoxon signed rank test used for analysis CWS results. ${ }^{*} \mathrm{P}<0.05$ considered significant

significantly less likely to report a CWS score consistent with severe distress than those without (22\% vs $77.8 \%)$ at baseline $(\mathrm{T} 1)\left(\chi^{2}(1)=7.670, \mathrm{p}=0.006\right)$. Notably, those with a family history of cancer in a first degree relative reported less anxiety at baseline with scores 2.4 points lower in HADS, and 7.78 points lower on the STAI-6 questionnaire compared to those with no family history, although this was not significant. No other significant relationships were observed between psychological outcomes and age; family history of NF1, any cancer, or breast cancer; and screening results including recall and biopsy. 


\section{Satisfaction, experience and barriers related to breast screening}

Satisfaction with breast screening procedures was high with the majority $\geq 80 \%$ (R1) and $\geq 78 \%$ (R2) 'quite' or 'very' satisfied with MRI, mammogram and ultrasound. Most participants $(\geq 76 \%)$ also rated their experience of all screening modalities as 'not at all' or only 'a little' embarrassing, uncomfortable, painful, worrying, or distressing. However only around half of these women considered MRI, mammogram and ultrasound to be 'moderately' to 'extremely' reassuring after receiving their results from breast screening (Table 5). Indeed, a small proportion of women with NF1 found MRI, mammogram and ultrasound 'moderately' to 'extremely' worrying, with $\geq 29 \%$ rating mammogram as 'moderately' to 'extremely' uncomfortable or painful. Despite this worry, discomfort and pain, most women in this study ( $\geq 85 \%$ ) did not rate physical discomfort, finding the scans distressing, or transport as a major barrier to attending future MRI, mammogram or ultrasound screening; with the exception of physical discomfort in mammograms where $>30 \%$ of women in R2 felt this was a 'moderate' barrier to future screening. A few participants (15-20\%) in R1 and $\mathrm{R} 2$ also reported 'taking time from obligations' was a potential barrier and $\geq 30 \%$ of women in $\mathrm{R} 2$ reported 'fear of results' as a potential barrier to attending future MRI, mammogram and ultrasound.

\section{Second round breast screening}

Of those 22 women who attended R1 and R2 breast screening, 19 completed R2 validated scales (HADS, HQ, STAI-6 and CWS). No significant differences in the post-test result scores, for the 16 participants who completed both first round (T3/4) and second round (R2), were detected for any of the validated scales. This is despite high STAI- 6 anxiety levels (mean STAI-6 scores 36.45 $\pm 15.1 \mathrm{~T} 3 / 4 \mathrm{v} 43.86 \pm 17.0$ R2) $(\mathrm{t}=-1.408, \mathrm{df}=15, \mathrm{p}=0.180)$, and slightly increased HADS anxiety scores in R2 as compared to T3/T4 (mean HADS scores $7.31 \pm 4.9 \mathrm{~T} 3 / 4 \times 7.50 \pm 5.8 \mathrm{R} 2)(\mathrm{t}=-0.337$, $\mathrm{df}=15, \mathrm{p}=0.741)$ Slightly increased HADS anxiety levels were also seen in the 19 participants who completed questionnaires at T1 and R2 (T1 mean HADS anxiety 7.21 \pm 4.1 $\mathrm{T} 1 \mathrm{v} 7.68 \pm 5.7 \mathrm{~T} 2),(\mathrm{t}=0.472, \mathrm{df}=18, \mathrm{p}=0.642)$ (Table 3$)$. There was no association between having increased anxiety levels (borderline/abnormal) at T1 or T3/T4 and attending second round screening, although numbers were small (Fisher exact test $\mathrm{T} 1 \mathrm{p}=0.432, \mathrm{~T} 3 / 4 \mathrm{p}=1.00$ ).

As for R1, the majority of R2 participants who completed ad hoc questions related to experience and barriers were 'quite' or 'very' satisfied with their experience of MRI, mammogram and ultrasound (R2: $77.8 \%, 100 \%$ and $100 \%$ respectively). Of interest, comparison of individual results for women who completed both R1 (T3) and R2 surveys demonstrated more women found MRI $(n=15)$, mammogram $(n=9)$ and ultrasound $(n=12)$ 'very' to 'extremely' reassuring during $\mathrm{R} 2$ compared to $\mathrm{R} 1$ (R2: $66,7 \%, 70 \%$ and $60 \%$ vs R1: $20 \%, 14.3 \%, 28.6 \%$ respectively). Other experiences and barriers appeared to be similar between R1 and R2 (Table 5).

\section{Screening decliners}

Of the original 28 who consented to participate in this substudy, two did not complete R1, and an additional five did not complete R2. Reasons for not attending were ascertained during routine follow up from six out of seven participants who did not complete R1 or R2. This included difficulty attending appointments in general (due to needing time off work, clashes with work schedules, anxiety related to MRI screening or long distances to travel) $(n=2)$, temporary difficulty attending the clinic (e.g., due to recovery from surgery, being unwell or pregnant) $(n=3)$ and due to an administrative error meaning that the participant was not automatically booked in for their R2 screening $(n=1)$. The four participants in the latter 2 groups were interested in re-joining the risk management clinic and have since been booked for R2 screening. The remaining participant deferred screening by 3 months, then was uncontactable. Four participants completed the decliner questionnaire (Q1D), but there was insufficient data for analysis.

\section{Discussion}

To our knowledge, this is the first study to examine the psychological impact of breast cancer screening in young women with NF1. Preliminary findings suggest the majority of women with NF1 do not suffer adverse effects due to screening and most experience satisfaction with surveillance through a breast cancer risk management clinic. Indeed, stable or decreased measures of psychological distress were associated with breast cancer surveillance. Individual scores did demonstrate a proportion of women with NF1 exhibit clinical levels of anxiety, cancer worry, and worsening behaviours, yet the ratio of individuals in each category did not change significantly over the course of breast cancer surveillance time points and these findings are most likely not related to the screening process. Despite the lack of a negative psychological impact of breast screening on young women with NF1, only half of the respondents who undertook the first round of breast screening reported that they were reassured by their results (regardless of the screening modality), however most women who attended a second round of breast screening were reassured. Surprisingly, 


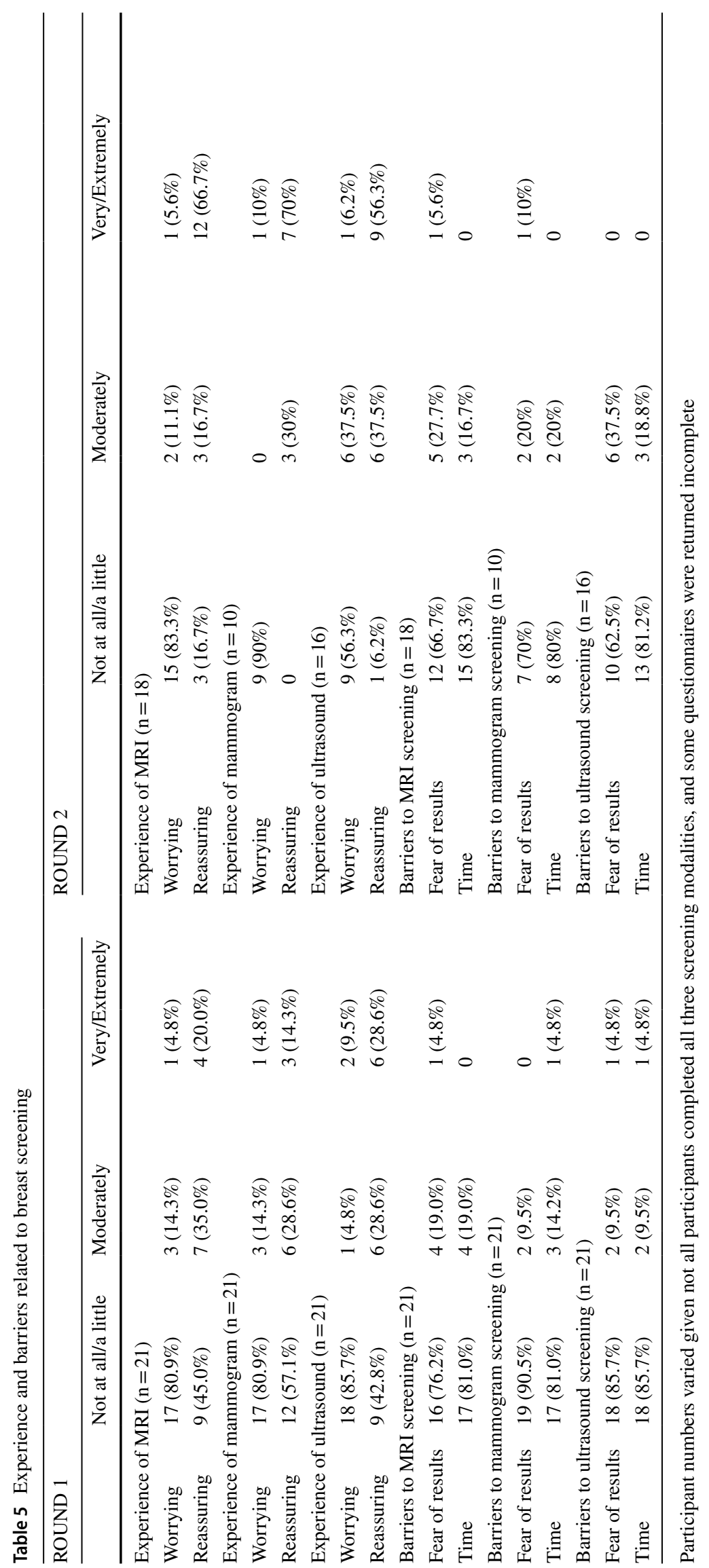


recall and biopsy were not associated with increased psychological distress.

Previous studies have reported a short-term increase in psychological distress in women undergoing breast cancer screening $[40,47,58]$. However, in the current study, similar to other high-risk populations (e.g., individuals who carry a $B R C A 1, B R C A 2$ or TP53 pathogenic variant, or with a family history of breast and pancreatic cancer) undertaking MRI-based cancer surveillance, stable or decreased measures of psychological distress were reported [39, 48, 59, 60]. In this study, some women with NF1 did demonstrate higher levels of anxiety and cancer worry prior to breast cancer screening, compared to other MRI-based screening studies $[39,40,48,59,60]$, which persisted over the course of the study. Although the anxiety measures used in this study have not been commonly used in population studies in NF1, the higher rate of anxiety in NF1 is consistent with previous literature $[18,21]$. Of interest, women with NF1 have previously been shown to display increased psychosocial, physical morbidity and decreased quality of life (QoL) $[13,20,21,61]$ in contrast to other studies of women at increased breast cancer risk, where QoL scores are closer to the general population [38]. We propose that heightened anxiety and cancer worry may be related to the reported increased incidence of NF1-specific cognitive and mental health issues, as well as the poorer $\mathrm{QoL}$ associated with the condition [62-66]. Therefore, the possible physical, cognitive and psychological impacts of NF1 may indicate that at least some women with NF1 already have a sustained high level of psychological distress that may have contributed to the lack of additional negative psychological impact associated with breast screening [40, 47, 58].

Although several women enrolled in this study were subjected to recall and biopsy, these investigations were not associated with further impact on psychological measures. This finding is reflected in other small studies, where recall after MRI was not associated with increased anxiety in a small cohort of women with a family history of breast cancer [40], nor were there adverse psychosocial outcomes in those requiring further investigations in a LFS whole body MRI screening study [67]. In contrast, previous studies of recall after MRI- or mammogram-based surveillance for other cohorts with cancer susceptibility syndromes demonstrated adverse psychological effects, which were transient $[39,68$, 69]. The very nature of NF1, as a tumour prone syndrome, may also help to explain this finding, given many would have already received medical interventions since childhood [7]. It is important to note that a true "baseline" was unattainable as participants completed their T1 questionnaire only after they provided consent to undergo breast screening.

Older age and having no family history of cancer in a first degree relative (FDR) were predictors of increased stress-related behaviour and cancer worry respectively.
International guidelines recommend extending screening to younger women with NF1 from age 30 [2-5]. Based on these findings, extending screening to younger women in Australia is unlikely to cause great psychological distress. In addition, having no family history of cancer in a FDR was a predictor of increased cancer worry, similar to a previous study in a high-risk breast cancer population [38]. This is in contrast to a study comparing women from the general population with those from hereditary breast and ovarian cancer (HBOC) families, which demonstrated that although all worried about developing breast or ovarian cancer, those from HBOC families worried more [70]. In a study of BRCA1 and BRCA2 pathogenic variant carriers, women were also more likely to elect for risk-reducing surgery if they had a direct experience of a loved one dying of cancer [71]. Additionally, studies of whole-body MRI screening for LFS were different again; the prevalence of clinically significant or borderline anxiety and depression did not differ between those with and without a strong family cancer history [67]. We therefore hypothesize that at the time of recruitment, learning of their increased risk was alarming for cancer-threat naïve patients. In future studies, it would be useful to consider participant knowledge of cancer risk prior to recruitment.

Women in this study also described some negative aspects to breast cancer screening, despite high satisfaction with the screening process. Approximately a third of women reported experiencing some 'discomfort' or 'pain' during mammogram, as reported previously [38, 40]. In addition, some respondents reported 'fear of their results' was a potential barrier. These are important findings as logistical difficulties in attending appointments, increased levels of cancer worry, and screening distress could impair adherence to surveillance and thus offset the benefits of screening [36, 37, 58, 72]. Indeed, several women did not return to screening in the second year, requiring prompting to attend. This was despite an appointment being automatically booked into the risk management clinic, and the provision of a phone call to coordinate screening bookings one month prior to the clinic. Decreased uptake of health monitoring and management has been reported in adults with NF1 [12] and may explain this finding. As women with NF1 also appear to be more prone to anxiety and cancer worry in general, this highlights an area of care requiring sensitive support, additional resources, and further exploration in the future. Strategies such as utilization of brief, tailored patient-reported experience and outcome measures (PREMs and PROMs) could be used to identify those at risk of psychological distress. In addition, early educational interventions to alleviate and manage patient concerns and expectations, and to promote adherence to screening could be introduced as recommended previously [34, 35, 40, 73].

The limitations of this pilot study include the recruitment of only a small number of participants. A post-hoc power 
analysis revealed that sample sizes of 65 (HADS-anxiety), 35 (HADS-depression), 79 (CWS), 68 (HQ) and 660 (STAI6) would be needed to detect a 1-point difference between pairs $(\mathrm{T} 1, \mathrm{~T} 3)$ at a power of $80 \%$ and a level of significance of $5 \%$. Bias is also likely due to the self-selection of the patient cohort who may be more likely to be healthy; have higher neurocognitive abilities; more able to cope; and already engaged with a specialist NF1 clinic. In addition, some participants completed screening earlier than the time points outlined in the study design, which may have skewed results. Some potential confounders were not considered including severity and psychosocial impact of NF1, physical disability, health activation and pre-existing anxiety and depression, which may have confounded comparison with other high-risk populations. In addition, a number of women were completing their T4 or R2 questionnaires at the beginning of the Covid-19 pandemic, which has been associated with an increase in anxiety and depression within the Australian population $[74,75]$. As the numbers for first and second round screening were small, and participant drop out was an issue, these findings need to be confirmed.

The results of this study identify a number of clinical implications that could be considered when counselling young women with NF1 prior to and during early breast cancer surveillance. Some women may exhibit pre-existing clinical levels of psychological distress; cancer-naïve and older women (35-50 years) may experience higher levels of cancer worry; and other participants may have had negative experiences of cancer within the family. In addition, some women may experience barriers to attendance, reduced adherence to recurrent breast screening, or lower health activation known to be exhibited by individuals with NF1. To support these women, the number of appointments should be minimised where possible (e.g., combining screening). In addition, promotion of patient engagement through genetic counselling, access to psychological services, plus targeted educational resources may encourage patients to harbor more realistic expectations of the screening process and encourage them to adhere to subsequent rounds of breast screening.

\section{Conclusion}

In this study of the psychological impact of breast screening in young women with NF1 we demonstrated no significant psychological impact of early breast screening in an established risk management clinic and high levels of satisfaction with screening. Although, some individuals exhibited clinical levels of anxiety, cancer worry and stress-related behavioural change, these were not impacted by breast screening. These findings are important for future research planning and have clinical implications for the management of breast cancer risk in young women with NF1. Clinician awareness surrounding the high levels of baseline anxiety and cancer worry, and exploration of potential barriers with patients (fear of results, screening discomfort, transport and logistical difficulties) may alleviate patient concerns and promote better engagement in care. In addition, the development of information to address potential barriers and easy to administer PREMs and PROMs may promote adherence to screening and help clinicians to identify those who might benefit from increased support due to increased psychological concerns throughout the testing process.

Supplementary Information The online version contains supplementary material available at https://doi.org/10.1007/s10689-021-00259-9.

Acknowledgements We are most grateful to the patient participants for their time and willingness to contribute this study. In addition, thank you to Dr Georgina Luscombe and Dr Rachel O'Connell for providing statistical support, Dr Ranjani Reddy and Dr Sarah Choi and the North Shore Radiology and Nuclear Medicine team for facilitating breast screening, and Dr Jeffery Thompson, who collated the demographic information. In addition, we appreciate the input from Dr Mandy Ballinger and the SMOC+ study investigators for useful discussions regarding screening protocols and psychosocial assessment tools.

Author contributions All authors contributed to the study conception and design. Material preparation, data collection and analysis were performed by AC, Dr MW, Dr LT, Dr RK, SE and Dr JF. The first draft of the manuscript was written jointly by AC, Dr RK, SE and Dr JF. All authors commented on previous versions of the manuscript. All authors read and approved the final manuscript.

Funding This research was supported by a grant procured from the Children's Tumour Foundation and The Honourable Brad Hazzard Minister for Health and Medical Research.

\section{Declarations}

Conflict of interest The authors declare that they have no conflict of interest.

Consent to participate Informed consent was obtained from all individuals included in the study.

Consent for publication Participants consented to publication of deidentified information on the consent to participate. Only de-identified information included.

Ethical approval Approval was obtained from the Human Research Ethics Committee of Northern Sydney Local Health District. Approval was granted by the ethics committee on 18 June 2018: 2020ETH00762. The procedures used in this study adhere to the tenets of the Declaration of Helsinki.

\section{References}

1. Seminog OO, Goldacre MJ (2015) Age-specific risk of breast cancer in women with neurofibromatosis type 1 . Br J Cancer 112(9):1546-1548. https://doi.org/10.1038/bjc.2015.78 
2. Suarez-Kelly LP, Yu L, Kline D, Schneider EB, Agnese DM, Carson WE (2019) Increased breast cancer risk in women with neurofibromatosis type 1: a meta-analysis and systematic review of the literature. Hered Cancer Clin Pract. https://doi.org/10.1186/ s13053-019-0110-z

3. Uusitalo E, Kallionpaa RA, Kurki S et al (2017) Breast cancer in neurofibromatosis type 1: overrepresentation of unfavourable prognostic factors. Br J Cancer 116(2):211-217. https://doi.org/ 10.1038/bjc.2016.403

4. Daly MB, Pilarski R, Berry M et al (2017) NCCN Guidelines Insights: Genetic/Familial High-Risk Assessment: breast and ovarian, version 2.2017. J Natl Compr Canc Netw 15(1):9-20. https://doi.org/10.6004/jncen.2017.0003

5. Stewart DR, Korf BR, Nathanson KL, Stevenson DA, Yohay K (2018) Care of adults with neurofibromatosis type 1: a clinical practice resource of the American College of Medical Genetics and Genomics (ACMG). Genet Med 20(7):671-682. https://doi. org/10.1038/gim.2018.28

6. NSW Cancer Institute (2020) NF1 (Neurofibromatosis type 1)risk management. vol. 14/11, NSW Government, https://eviq.org. $\mathrm{au} /$ cancer-genetics/adult/risk-management/752-nf1-neurofibro matosis-type-1-risk-manageme. Accessed 3 Dec 2020

7. Ferner RE, Huson SM, Thomas N et al (2007) Guidelines for the diagnosis and management of individuals with neurofibromatosis 1. J Med Genet 44(2):81-88. https://doi.org/10.1136/jmg.2006. 045906

8. Madanikia SA, Bergner A, Ye X, Blakeley JON (2012) Increased risk of breast cancer in women with NF1. Am J Med Genet A 158(12):3056-3060. https://doi.org/10.1002/ajmg.a.35550

9. Uusitalo E, Rantanen M, Kallionpää RA et al (2016) Distinctive cancer associations in patients with neurofibromatosis type 1 . J Clin Oncol 34(17):1978-1986. https://doi.org/10.1200/JCO.2015. 65.3576

10. Easton DF, Ponder MA, Huson SM, Ponder BA (1993) An analysis of variation in expression of neurofibromatosis (NF) type 1 (NF1): evidence for modifying genes. Am J Hum Genet 53(2):305-313

11. (1988) Neurofibromatosis. Conference statement. National Institutes of Health Consensus Development Conference. Arch Neurol 45(5):575-578. https://pubmed.ncbi.nlm.nih.gov/3128965/

12. Crawford H, Barton B, Wilson $M$ et al (2015) The impact of neurofibromatosis type 1 on the health and wellbeing of Australian adults. J Genet Couns 24(6):931-944. https://doi.org/10.1007/ s10897-015-9829-5

13. Hirbe AC, Gutmann DH (2014) Neurofibromatosis type 1: a multidisciplinary approach to care. Lancet Neurol 13(8):834-843. https://doi.org/10.1016/S1474-4422(14)70063-8

14. Templer AK, Titus JB, Gutmann DH (2013) A neuropsychological perspective on attention problems in neurofibromatosis type 1 . $\mathrm{J}$ Atten Disord 17(6):489-496. https://doi.org/10.1177/1087054711 433422

15. Ferner R, Thomas M, Mercer G et al (2017) Evaluation of quality of life in adults with neurofibromatosis 1 (NF1) using the Impact of NF1 on Quality Of Life (INF1-QOL) questionnaire. Health Qual Life Outcomes. https://doi.org/10.1186/s12955-017-0607-y

16. Ferner RE (2011) Neurofibromatoses in clinical practice. Springer, London

17. Granström S, Langenbruch A, Augustin M, Mautner V-F (2012) Psychological burden in adult neurofibromatosis type 1 patients: impact of disease visibility on body image. Dermatology 224(2):160-167. https://doi.org/10.1159/000337548

18. Cohen JS, Levy HP, Sloan J, Dariotis J, Biesecker BB (2015) Depression among adults with neurofibromatosis type 1: prevalence and impact on quality of life. Clin Genet 88(5):425-430. https://doi.org/10.1111/cge.12551
19. Ferner RE, Hughes RAC, Weinman J (1996) Intellectual impairment in neurofibromatosis 1. J Neurol Sci 138(1-2):125-133. https://doi.org/10.1016/0022-510X(96)00022-6

20. Sanagoo A, Jouybari L, Koohi F, Sayehmiri F (2019) Evaluation of QoL in neurofibromatosis patients: a systematic review and meta-analysis study. (Report). BMC Neurol. https://doi.org/10. 1186/s12883-019-1338-y

21. Hamoy-Jimenez G, Kim R, Suppiah S, Zadeh G, Bril V, Barnett C (2020) Quality of life in patients with neurofibromatosis type 1 and 2 in Canada. Neuro-Oncol Adv 2(Supplement_1):i141-i149. https://doi.org/10.1093/noajnl/vdaa003

22. Sharif S, Moran A, Huson SM et al (2007) Women with neurofibromatosis 1 are at a moderately increased risk of developing breast cancer and should be considered for early screening. J Med Genet 44(8):481. https://doi.org/10.1136/jmg.2007.049346

23. Gottfried ON, Viskochil DH, Couldwell WT (2010) Neurofibromatosis type 1 and tumorigenesis: molecular mechanisms and therapeutic implications. Neurosurg Focus 28(1):E8. https://doi. org/10.3171/2009.11.Focus09221

24. Pradhan D, Kaur N, Gami A, Hura KS, Garg G, Mohanty SK (2017) Neurofibromatosis and breast cancer: do we need to revise the mammographic screening schedule in patients of neurofibromatosis? J Cancer Res Ther 13(3):583-585. https://doi.org/ 10.4103/0973-1482.196761

25. Yap YS, Munusamy P, Lim C et al (2018) Breast cancer in women with neurofibromatosis type 1 (NF1): a comprehensive case series with molecular insights into its aggressive phenotype. Breast Cancer Res Treat 171(3):719-735. https://doi.org/10.1007/ s10549-018-4851-6

26. Maani N, Westergard S, Yang J et al (2019) NF1 patients receiving breast cancer screening: insights from the Ontario High Risk Breast Screening Program. Cancers (Basel). https://doi.org/10. 3390/cancers 11050707

27. Evans DGR, Kallionpaa RA, Clementi M et al (2020) Breast cancer in neurofibromatosis 1: survival and risk of contralateral breast cancer in a five country cohort study. Genet Med 22(2):398-406. https://doi.org/10.1038/s41436-019-0651-6

28. Da Silva AV, Rodrigues FR, Pureza M, Lopes VGS, Cunha KS (2015) Breast cancer and neurofibromatosis type 1: a diagnostic challenge in patients with a high number of neurofibromas. BMC Cancer 15(1): 183. https://doi.org/10.1186/s12885-015-1215-z

29. Evans DG, O'Hara C, Wilding A et al (2011) Mortality in neurofibromatosis 1: in North West England: an assessment of actuarial survival in a region of the UK since 1989. Eur J Hum Genet 19(11):1187-1191. https://doi.org/10.1038/ejhg.2011.113

30. Crawford HA, Barton B, Wilson MJ et al (2016) Uptake of health monitoring and disease self-management in Australian adults with neurofibromatosis type 1: strategies to improve care. Clin Genet 89(3):385-391. https://doi.org/10.1111/cge.12627

31. Hibbard J, Greene J (2013) What the evidence shows about patient activation: better health outcomes and care experiences; Fewer data on costs. Health Aff 32:207-214

32. Nelson HD, Pappas M, Cantor A, Griffin J, Daeges M, Humphrey L (2016) Harms of breast cancer screening: systematic review to update the 2009 US Preventive Services Task Force Recommendation. Ann Intern Med 164(4):256. https://doi.org/10.7326/ M15-0970

33. Scaf-Klomp W, Sanderman R, van de Wiel HB, Otter R, van Den Heuvel WJ (1997) Distressed or relieved? Psychological side effects of breast cancer screening in The Netherlands. J Epidemiol Community Health 51(6):705. https://doi.org/10.1136/jech.51.6. 705

34. Brain K, Henderson BJ, Tyndel S et al (2008) Predictors of breast cancer-related distress following mammography screening in younger women on a family history breast screening programme. 
Psychooncology 17(12):1180-1188. https://doi.org/10.1002/pon. 1355

35. Independent Uk Panel On Breast Cancer S (2012) The benefits and harms of breast cancer screening: an independent review. The Lancet 380(9855):1778-1786. https://doi.org/10.1016/S01406736(12)61611-0

36. van Dooren S, Seynaeve C, Rijnsburger AJ et al (2005) Exploring the course of psychological distress around two successive control visits in women at hereditary risk of breast cancer. Eur J Cancer 41(10):1416-1425. https://doi.org/10.1016/j.ejca.2005.03.020

37. Kash KM, Holland JC, Halper MS, Miller DG (1992) Psychological distress and surveillance behaviors of women with a family history of breast cancer. J Natl Cancer Inst 84(1):24-30. https:// doi.org/10.1093/jnci/84.1.24

38. Rijnsburger AJ, Essink-Bot ML, van Dooren S et al (2004) Impact of screening for breast cancer in high-risk women on healthrelated quality of life. Br J Cancer 91(1):69-76. https://doi.org/ 10.1038/sj.bjc.6601912

39. Spiegel TN, Esplen MJ, Hill KA, Wong J, Causer PA, Warner E (2011) Psychological impact of recall on women with BRCA mutations undergoing MRI surveillance. Breast 20(5):424-430. https://doi.org/10.1016/j.breast.2011.04.004

40. Hutton J, Walker LG, Gilbert FJ et al (2011) Psychological impact and acceptability of magnetic resonance imaging and X-ray mammography: the MARIBS Study. Br J Cancer 104(4):578-586. https://doi.org/10.1038/bjc.2011.1

41. Pashayan N, Pharoah P, Tabár L et al (2011) Validation of a modelling approach for estimating the likely effectiveness of cancer screening using cancer data on prevalence screening and incidence. Cancer Epidemiol 35(2):139-144. https://doi.org/10. 1016/j.canep.2010.07.012

42. Kurian AW, Sigal BM, Plevritis SK (2010) Survival analysis of cancer risk reduction strategies for BRCA1/2 mutation carriers. J Clin Oncol 28(2):222-231. https://doi.org/10.1200/JCO.2009.22. 7991

43. Saadatmand S, Obdeijn IM, Rutgers EJ et al (2015) Survival benefit in women with BRCA1 mutation or familial risk in the MRI screening study (MRISC). Int J Cancer 137(7):1729-1738. https:// doi.org/10.1002/ijc.29534

44. Evans DG, Harkness EF, Howell A et al (2016) Intensive breast screening in BRCA2 mutation carriers is associated with reduced breast cancer specific and all cause mortality. Hered Cancer Clin Pract. https://doi.org/10.1186/s13053-016-0048-3

45. Warner E, Plewes DB, Hill KA et al (2004) Surveillance of BRCA1 and BRCA2 mutation carriers with magnetic resonance imaging, ultrasound, mammography, and clinical breast examination. JAMA 292(11):1317-1325. https://doi.org/10.1001/jama. 292.11.1317

46. Gagnon P, Massie MJ, Kash KM et al (1996) Perception of breast cancer risk and psychological distress in women attending a surveillance program. Psychooncology 5(3):259-269. https://doi.org/ 10.1002/(SICI)1099-1611(199609)5:3\%3c259::AID-PON204\% 3e3.0.CO;2-O

47. Reichelt J, Møller P, Heimdal K, Dahl A (2008) Psychological and cancer-specific distress at 18 months post-testing in women with demonstrated BRCA1 mutations for hereditary breast/ovarian cancer. Fam Cancer 7(3):245-254. https://doi.org/10.1007/ s10689-008-9182-z

48. Konings IC, Sidharta GN, Harinck F et al (2016) Repeated participation in pancreatic cancer surveillance by high-risk individuals imposes low psychological burden. Psychooncology 25(8):971978. https://doi.org/10.1002/pon.4047

49. Zigmond AS, Snaith RP (1983) The hospital anxiety and depression scale. Acta Psychiatr Scand 67(6):361-370. https://doi.org/ 10.1111/j.1600-0447.1983.tb09716.x
50. Marteau TM, Bekker H (1992) The development of a six-item short-form of the state scale of the Spielberger State-Trait Anxiety Inventory (STAI). Br J Clin Psychol 31(3):301-306. https://doi. org/10.1111/j.2044-8260.1992.tb00997.x

51. Spielberger C, Gorusch R, Lushene R (1970) Manual for the StateTrait anxiety. Consulting Psychologists Press, California

52. Spielberger C, Gorusch R, Lushene R (1983) Manual for the StateTrait anxiety inventory-form Y. Consulting Psychologists Press, Palo Alto, CA

53. Custers EJA, Van Den Berg WS, Van Laarhoven MHW, Bleiker AEM, Gielissen MMF, Prins BJ (2014) The Cancer Worry Scale: detecting fear of recurrence in breast cancer survivors. Cancer Nurs 37(1):E44-E50. https://doi.org/10.1097/NCC.0b013e3182 $813 \mathrm{a} 17$

54. Douma KF, Aaronson NK, Vasen HF et al (2010) Psychological distress and use of psychosocial support in familial adenomatous polyposis. Psychooncology 19(3):289-298. https://doi.org/10. 1002/pon. 1570

55. Walker DLG, Cordiner CM, Gilbert FJ et al (1994) How distressing is attendance for routine breast screening? Psychooncology 3:229-304. https://doi.org/10.1002/pon.2960030406

56. Ballinger ML, Best A, Mai PL et al (2017) Baseline surveillance in Li-Fraumeni syndrome using whole-body magnetic resonance imaging: a meta-analysis. JAMA Oncol 3(12):1634-1639. https:// doi.org/10.1001/jamaoncol.2017.1968

57. Harris PA, Taylor R, Thielke R, Payne J, Gonzalez N, Conde JG (2009) Research electronic data capture (REDCap) — a metadatadriven methodology and workflow process for providing translational research informatics support. J Biomed Inform 42(2):377381. https://doi.org/10.1016/j.jbi.2008.08.010

58. O'Neill S, Rubinstein W, Sener S et al (2009) Psychological impact of recall in high-risk breast MRI screening. Breast Cancer Res Treat 115(2):365-371. https://doi.org/10.1007/ s10549-008-0140-0

59. Bancroft EK, Saya S, Page EC et al (2019) Psychosocial impact of undergoing prostate cancer screening for men with BRCA1 or BRCA2 mutations. BJU Int 123(2):284-292. https://doi.org/10. 1111/bju. 14412

60. McBride KA, Ballinger ML, Schlub TE et al (2017) Psychosocial morbidity in TP53 mutation carriers: is whole-body cancer screening beneficial? Fam Cancer 16(3):423-432. https://doi.org/ 10.1007/s10689-016-9964-7

61. Daphne LW, Kelly BS, Sonia E et al (2012) Emotional functioning of patients with neurofibromatosis tumor suppressor syndrome. Genet Med 14(12):977. https://doi.org/10.1038/gim.2012.85

62. Doser K, Andersen EW, Kenborg L et al (2020) Clinical characteristics and quality of life, depression, and anxiety in adults with neurofibromatosis type 1: a nationwide study. Am J Med Genet A 182(7):1704-1715. https://doi.org/10.1002/ajmg.a.61627

63. Nutakki K, Hingtgen CM, Monahan P, Varni JW, Swigonski NL (2013) Development of the adult PedsQL neurofibromatosis type 1 module: initial feasibility, reliability and validity. Health Qual Life Outcomes 11:21. https://doi.org/10.1186/1477-7525-11-21

64. Wolkenstein P, Zeller J, Revuz J, Ecosse E, Leplege A (2001) Quality-of-life impairment in neurofibromatosis type 1: a crosssectional study of 128 cases. Arch Dermatol 137(11):1421-1425. https://doi.org/10.1001/archderm.137.11.1421

65. Kodra Y, Giustini S, Divona L et al (2009) Health-related quality of life in patients with neurofibromatosis type 1 . A survey of 129 Italian patients. Dermatology 218(3):215-220. https://doi.org/10. $1159 / 000187594$

66. Page PZ, Page GP, Ecosse E, Korf BR, Leplege A, Wolkenstein $P$ (2006) Impact of neurofibromatosis 1 on Quality of Life: a cross-sectional study of 176 American cases. Am J Med Genet A 140(18):1893-1898. https://doi.org/10.1002/ajmg.a.31422 
67. Bancroft EK, Saya S, Brown E et al (2020) Psychosocial effects of whole-body MRI screening in adult high-risk pathogenic TP53 mutation carriers: a case-controlled study (SIGNIFY). J Med Genet 57(4):226-236. https://doi.org/10.1136/jmedg enet-2019-106407

68. Gilbert FJ, Cordiner CM, Affleck IR, Hood DB, Mathieson D, Walker LG (1998) Breast screening: the psychological sequelae of false-positive recall in women with and without a family history of breast cancer. Eur J Cancer 34(13):2010-2014. https://doi.org/ 10.1016/s0959-8049(98)00294-9

69. Lerman C, Rimer BK, Engstrom PF (1991) Cancer risk notification: psychosocial and ethical implications. J Clin Oncol 9(7):1275-1282. https://doi.org/10.1200/JCO.1991.9.7.1275

70. Chalmers K, Thomson K (1996) Coming to terms with the risk of breast cancer: perceptions of women with primary relatives with breast cancer. Qual Health Res 6(2):256-282. https://doi.org/10. $1177 / 104973239600600207$

71. Hesse-Biber S (2014) The genetic testing experience of BRCApositive women: deciding between surveillance and surgery. Qual Health Res 24(6):773-789. https://doi.org/10.1177/1049732314 529666

72. Rippinger N, Fischer C, Haun MW et al (2020) Cancer surveillance and distress among adult pathogenic TP53 germline variant carriers in Germany: a multicenter feasibility and acceptance survey. Cancer 126(17):4032-4041. https://doi.org/10.1002/cncr. 33004

73. Bond M, Pavey T, Welch K et al (2013) Psychological consequences of false-positive screening mammograms in the UK. Evid Based Med 18(2):54-61. https://doi.org/10.1136/eb-2012-100608

74. Hammarberg K, Tran T, Kirkman M, Fisher J (2020) Sex and age differences in clinically significant symptoms of depression and anxiety among people in Australia in the first month of COVID-19 restrictions: a national survey. BMJ Open 10(11):e042696. https:// doi.org/10.1136/bmjopen-2020-042696

75. Staples L, Nielssen O, Kayrouz R et al (2020) Rapid report 2: symptoms of anxiety and depression during the first 12 weeks of the Coronavirus (COVID-19) pandemic in Australia. Internet Interv 22:100351. https://doi.org/10.1016/j.invent.2020.100351

Publisher's Note Springer Nature remains neutral with regard to jurisdictional claims in published maps and institutional affiliations. 